\title{
在共聚物 P(GMA-co-MMA)侧链实现卟啉化合物的同步合成与键合
}

\author{
王飞宇高保娇* 王芯欣 \\ (中北大学化学工程系, 太原 030051)
}

\begin{abstract}
摘要：采用溶液聚合的方法, 先合成了甲基丙烯酸缩水甘油酯(GMA)与甲基丙烯酸甲酯(MMA)的线型共聚物 $\mathrm{P}(\mathrm{GMA}-c o-M M A)$; 然后通过环氧基团的开环成醚反应, 将对羟基苯甲醛(HBA)键合于共聚物的侧链上, 形成键 合有苯甲醛(BA)的聚合物 BA-P(GMA-co-MMA); 最后采用 Adler 方法, 以 BA-P(GMA-co-MMA)、苯甲醛及吡咯 为反应物, 在均相反应体系中, 成功地实现了苯基吓啉(PP)在共聚物 P(GMA-co-MMA)侧链的同步合成与键合, 制得了卟啉功能化的线型大分子 PP-P(GMA-co-MMA). 通过 FTIR 及 ${ }^{1} \mathrm{H}-\mathrm{NMR}$ 对功能大分子 PP-P(GMA-coMMA)的化学结构进行了表征, 对其谱学性能进行了研究, 较为深人地分析了其光物理行为. 实验结果表明, 大分 子 PP-P(GMA-co-MMA) 具有与四苯基卟啉(TPP)相同的电子吸收光谱与苂光发射光谱. 随着侧链上 PP 键合量 的增大, 大分子 PP-P(GMA-co-MMA)的 Soret 与 $Q$ 吸收带的峰强均不断增强, 而 $Q$ 发射带的强度则呈现先增大 后有所减弱的规律, 这是由大分子内的能量转移所致.
\end{abstract}

关键词：苯基吓啉; 甲基丙烯酸缩水甘油酯; 卟啉功能化的聚合物; 同步合成与键合; 光谱性能 中图分类号： O644; O631

\section{Simultaneous Synthesis and Linkage of Porphyrin onto Side Chain of Copolymer P(GMA-co-MMA)}

\author{
WANG Fei-Yu GAO Bao-Jiao* WANG Rui-Xin \\ (Department of Chemical Engineering, North University of China, Taiyuan 030051, P. R. China)
}

\begin{abstract}
The copolymerization of glyceryl methacrylate (GMA) and methyl methacrylate (MMA) was conducted in a solution polymerization system resulting in the formation of copolymer P (GMA-co-MMA). Parahydroxybenzaldehyde was then bound to the side chains of $\mathrm{P}(\mathrm{GMA}-$ co-MMA) via ring-opening and ether-forming reactions of the epoxy groups on P(GMA-co-MMA). A benzaldehyde (BA)-bound copolymer BA-P(GMA-co-MMA) was thus prepared. The simultaneous synthesis and linkage of the porphyrin onto the side chains of $\mathrm{P}(\mathrm{GMA}-\mathrm{co}-\mathrm{MMA})$ was realized successfully using the Adler method in a homogeneous system. Benzaldehyde, BA-P(GMA-co-MMA), and pyrrole were used as reactants, resulting in the porphyrin (PP)-functionalized line-type macromolecule PP-P(GMA-co-MMA). The chemical structure of PP-P(GMA-co-MMA) was characterized by Fourier transform infrared (FTIR) spectroscopy and nuclear magnetic resonance ( $\left.{ }^{1} \mathrm{H}-\mathrm{NMR}\right)$ spectroscopy. The spectral properties of PP-P(GMA-co-MMA) were studied and its photophysical behavior was investigated in depth. Experimental results show that PP-P(GMA-co-MMA) has the same electronic absorption spectrum and fluorescence emission spectrum as tetraphenyl porphyrin. In the electronic absorption spectra of PP-P (GMA-co-MMA) both the Soret absorption band and the $Q$ absorption band strengthen as the amount of bound porphyrin increaseds. The $Q$ emission band intensity of PP-P(GMA-co-MMA) also first increases with the increase of the amount of bound porphyrin until to a maximum, and then decreases if more porphyrin units are present. Intramolecular energy transfer of the macromolecule PP-P(GMA-co-MMA) should be responsible for this behavior.
\end{abstract}

Key Words: $\quad$ Phenyl porphyrin; Glyceryl methacrylate; Porphyrin-functionalized polymer; Synchronistic synthesis and linkage; Spectrum property

Received: August 15, 2008; Revised: October 10, 2008; Published on Web: November 18, 2008.

*Corresponding author. Email: gaobaojiao@126.com; Tel: +8635-3921414.

山西省自然科学基金(2008021013)资助项目

(C) Editorial office of Acta Physico-Chimica Sinica 
卟啉是一类具有共轭骨架的大环化合物, 在自 然界和生命体中广泛存在. 卟啉化合物独特的化学 结构, 赋予其特殊的生理活性与物理化学性能, 在目 前的材料科学中, 模拟天然卟啉的结构与功能, 合成 结构与性能各异的卟啉与金属卟啉, 已经形成了一 个极为活跃的研究领域. 卟啉化合物在医学(光动力 治疗)、生物化学(分子识别)、材料化学(光电材料)、 合成化学(仿生催化)、分析化学(光度分析及传感 器)、能源科学(太阳能转换) 等 ${ }^{[1-7]}$ 多个领域都有着重 要的应用.

天然金属卟啉是在特定的天然大分子(多肽)营 造的氛围中发挥其特性的, 同时也受到了高分子微 环境的保护, 使其具有化学稳定性 ${ }^{[8,9]}$. 而仿生制备的 小分子吓啉或金属卟啉, 则缺乏化学稳定性, 另一方 面又不具备加工性能, 使其应用受到一定的限制. 比 如作为有机电致发光材料的组份之一, 小分子吓啉 只能掺杂在聚合物基质中使用, 既容易发生浓度猝 灭, 也影响材料的力学性能 ${ }^{[1,11]}$. 实现卟啉或金属叶 啉的高分子化或高聚物的卟啉功能化, 既可为卟啉 化合物营造一个类似多肽的微环境, 以提高其化学 稳定性, 充分发挥其特性; 又可使吓啉化合物也具 有了高分子材料的良好加工性能, 比如成膜、成球性 能等, 从而可极大地拓展卟啉化合物的应用范围, 提 升其应用效能. 因此, 经过分子设计并通过一定的化 学途径, 实现高聚物的吓啉功能化, 将成为材料科学 中具有重大科学意义的研究课题. 但迄今为止, 对高 分子吓啉功能化的研究尚很少 ${ }^{[12,13]}$, 在卟啉功能材料 的相关研究中, 大多采用在基质中掺杂小分子卟啉

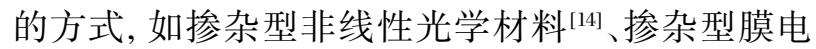

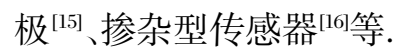

本文采用在共聚物 P(GMA-co-MMA)侧链同步 合成与键合卟啉的方法, 制得了吓啉功能化的大分 子 PP-P(GMA-co-MMA), 并研究了该功能聚合物的 谱学性能, 较深人地分析了光物理行为.

\section{1 实验部分}

\section{1 试剂与仪器}

甲基丙烯酸缩水甘油酯(GMA, 苏州南航化工有 限公司), 分析纯, 使用前经减压蒸馏提纯; 甲基丙烯 酸甲酯(MMA, 天津市瑞金特化学品有限公司), 分析 纯, 使用前经减压蒸馏提纯; 偶氮二异丁腈(AIBN, 上海化学试剂厂), 分析纯, 乙醇重结晶纯化; 对差基 苯甲醛(HBA, 天津市科密欧化学试剂开发中心), 分
析纯; 苯甲醛(BA, 天津市天力化学试剂有限公司), 分析纯; 吡咯(上海科丰化学试剂有限公司), 化学纯; 水杨酸(天津市博迪化工有限公司), 分析纯; $N, N$-二 甲基甲酰胺(DMF, 天津市博迪化工有限公司), 分析 纯; 二甲基亚砜(DMSO, 天津市瑞金特化学品有限公 司), 分析纯; 其余试剂均为市售分析纯试剂.

美国 Perkin-Elmer1700 型傅立叶变换红外光谱 仪; 上海尤尼柯公司 UV-2602 型紫外-可见分光光 度计; 日本 HITACHI F-2500 苂光光谱仪; 瑞士 Bruker drx300 超导核磁共振仪.

\section{2 共聚物 P(GMA-co-MMA)的制备}

将 5.4 mL GMA、4.4 mL MMA(摩尔比为 1:1)以 及 $60 \mathrm{~mL}$ DMF 加人到装有搅拌器、温度计、回流冷 凝管的四口反应烧瓶中, 通人 $\mathrm{N}_{2}$ 排除空气 $30 \mathrm{~min}$, 加人引发剂 $\mathrm{AIBN}$ (单体质量的 $0.5 \%$ ), 在 $\mathrm{N}_{2}$ 保护下 于 $60{ }^{\circ} \mathrm{C}$ 恒温反应 $8 \mathrm{~h}$. 结束反应后, 以甲醇为沉淀 剂, 沉淀出聚合物, 用甲醇洗涤多次, 真空干燥, 即得 共聚物 P(GMA-co-MMA).

\section{3 共聚物 P(GMA-co-MMA) 与对羟基苯甲醛的 键合反应}

在装有电动摚拌器的 $100 \mathrm{~mL}$ 四口瓶中, 加人 $1.2 \mathrm{~g}$ 共聚物 $\mathrm{P}(\mathrm{GMA}-\mathrm{co}-\mathrm{MMA})$ 与 $20 \mathrm{~mL} \mathrm{DMF}$, 浸泡 $24 \mathrm{~h}$, 使共聚物充分溶解; 将 $18 \mathrm{~mL}$ 溶有 $1.0 \mathrm{~g}$ 对差 基苯甲醛(HBA)的 DMF 溶液及 $0.6 \mathrm{~g} \mathrm{NaHCO}_{3}$, 一并 加人四口瓶中, 在氮气保护下升温至 $100{ }^{\circ} \mathrm{C}$, 恒温反 应 $5 \mathrm{~h}$. 反应结束后, 立即用冰水浴将体系冷却至室 温, 用蒸馏水和甲醇的混合液为沉淀剂, 沉淀出产物 聚合物, 并用混合溶液冲洗多次, 真空干燥, 即得侧 链键合有 BA 的线型聚合物 BA-P(GMA-co-MMA).

\section{4 卟啉在共聚物 P(GMA-co-MMA) 侧链上的同 步合成与键合及产物的表征}

在装有搅拌器的 $100 \mathrm{~mL}$ 四口烧瓶中, 依次加 人 $15 \mathrm{~mL}$ 溶解有 $1.0 \mathrm{~g}$ 共聚物BA-P(GMA-co-MMA)的 DMF 、 $2.0 \mathrm{~mL}(0.02 \mathrm{~mol})$ 苯甲醛与 $6 \mathrm{~mL}$ DMSO, 再加 人 $1.5 \mathrm{~g}$ 水杨酸, 搅拌下加热至 $\left.130{ }^{\circ} \mathrm{C}\right)$. 用恒压滴液 漏斗逐滴加人 $1.4 \mathrm{~mL}(0.02 \mathrm{~mol})$ 吡咯, $15 \mathrm{~min}$ 内滴加完 毕, 于 $130{ }^{\circ} \mathrm{C}$ 的恒温条件下继续反应一定时间. 反应 结束后, 将混合物冷却至室温, 用蒸馏水和甲醇的混 合液沉淀出产物聚合物, 并用混合溶液洗涤多次, 直 到洗涤液中不再含有小分子卟啉为止(用分光光度 法检测), 真空干燥至恒重, 得到褐色的产物, 即为 侧链键合有苯基吓啉(PP)的线型共聚物 PP-P(GMA$c o-M M A)$. 通过控制反应时间, 制备了 PP 键合量不 
同的聚合物PP-P(GMA-co-MMA).

采用 $\mathrm{KBr}$ 压片法, 测定PP-P(GMA-co-MMA)的红 外光谱; 以氝代二甲基亚砜为溶剂, 测定PP- P(GMAco-MMA)的核磁共振氢谱, 表征其化学结构. 以DMF 为溶剂, 采用分光光度法 $(\lambda=416 \mathrm{~nm})$ 测定PP-P(GMA$c o-M M A)$ 分子链中PP的键合量 $(\mathrm{g} / 100 \mathrm{~g})$.

\subsection{PP-P(GMA-co-MMA) 光物理行为的研究}

\subsection{1 电子吸收光谱的测定}

分别配制四苯基吓啉(TPP) 和 PP-P(GMA-coMMA)的DMF溶液, 测定其电子吸收光谱, 考察卟啉 功能化聚合物 PP-P(GMA-co-MMA)的谱学特性.

\subsection{2 荧光发射光谱的测定}

分别配制 TPP 和 PP-P(GMA-co-MMA)的 DMF 溶液 (为避免分子间的浓度淬灭, 小分子 TPP 的浓 度及聚合物PP-P(GMA-co-MMA) 分子链中PP单元的 浓度均控制在 $10^{-6} \mathrm{~mol} \cdot \mathrm{L}^{-1}$ 数量级), 以 $\lambda=560 \mathrm{~nm}$ 的可 见光为激发光波, 测定它们的苂光发射光谱, 考察卟 啉功能化聚合物PP-P(GMA-co-MMA) 的光物理行 为特征.

\section{2 结果与讨论}

\section{1 在共聚物 P(GMA-co-MMA) 侧链实现卟啉同} 步合成与键合的化学过程

在 $\mathrm{NaHCO}_{3}$ 的催化作用下, $\mathrm{DMF}$ 溶液中的对差 基苯甲醛与线型共聚物 P(GMA-co-MMA)的环氧侧 基之间, 发生开环成醚反应, 从而生成侧链键合有苯 甲醛的线型聚合物BA-P(GMA-co-MMA); 在酸催化 剂作用下, DMF溶液中的苯甲醛、大分子BA-P(GMAco-MMA) 侧链上键合的苯甲醛与吡咯之间发生 Adler 反应, 实现苯基吓啉 $(\mathrm{PP})$ 在共聚物 P(GMAco-MMA)侧链的同步合成与化学键合, 从而制得了 卟啉功能化的线型聚合物 PP-P(GMA-co-MMA), 整

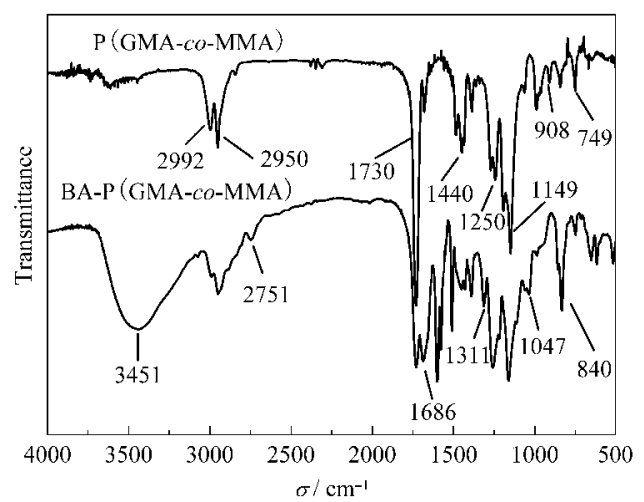

个化学过程如图式 1 所示.

(a)

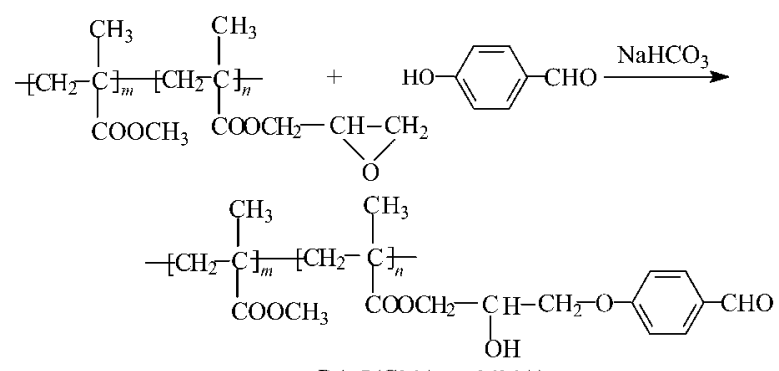

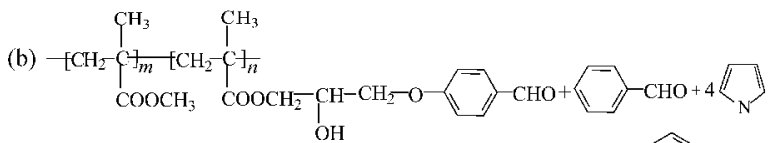

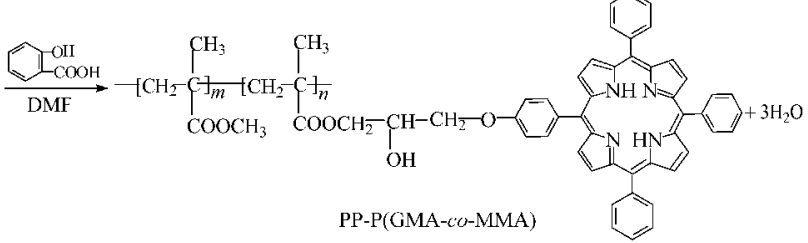

图式 1 苯基卟啉在共聚物 P(GMA-co-MMA)侧链同步合 成与键合的化学过程

Scheme1 Chemical process of synchronistic synthesis and bonding of PP on side chains of P(GMA-co-MMA)

(a) bonding of parahydroxybenzaldehyde on side chains of P(GMA-co-MMA); (b) synchronistically synthesizing and bonding porphyrin on side chains of $\mathrm{P}$ (GMA-co-MMA)

\subsection{PP-P(GMA-co-MMA) 的表征}

\subsubsection{PP-P(GMA-co-MMA)的红外光谱}

图1给出了 $\mathrm{P}(\mathrm{GMA}-c o-\mathrm{MMA}) 、 \mathrm{BA}-\mathrm{P}(\mathrm{GMA}-c o-$ MMA) 与 PP-P(GMA-co-MMA) 三种聚合物的红外 光谱.

在 P(GMA-co-MMA) 的红外光谱图中, 显示出 2 种甲基丙烯酸酯类单体单元的共同特征吸收峰: $1730 \mathrm{~cm}^{-1}$ 处的峰为酯羰基的特征吸收; 1250 与

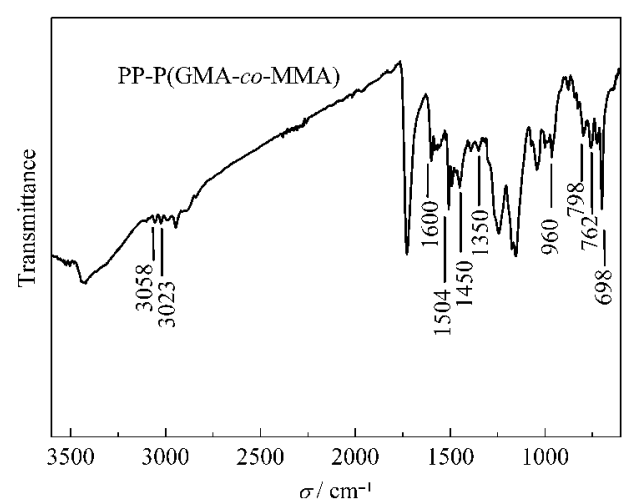

图 1 三种聚合物的红外光谱图

Fig.1 FTIR spectra of three copolymers 
$1149 \mathrm{~cm}^{-1}$ 处的峰分别为酯基中 $\mathrm{C}-\mathrm{O}-\mathrm{C}$ 键的不对 称与对称伸缩振动吸收; $2950 \mathrm{~cm}^{-1}$ 处的峰为则为主 链侧甲基的特征吸收; $2992 \mathrm{~cm}^{-1}$ 处的峰为主链亚甲 基的不对称伸缩振动吸收. $1440 \mathrm{~cm}^{-1}$ 处的峰则为 MMA 酯基中甲基的特征吸收. 最重要的是在 908 $\mathrm{cm}^{-1}$ 处显示出了 GMA 单元中环氧键的特征吸收.

在 BA-P(GMA-co-MMA) 的红外光谱图中, 908 $\mathrm{cm}^{-1}$ 处环氧键的特征吸收峰消失; 与此同时, 在 3451 与 $1311 \mathrm{~cm}^{-1}$ 处则出现了环氧键开环后生成的 仲羟基的振动吸收, 在 $1047 \mathrm{~cm}^{-1}$ 处出现了开环成醚 反应后所形成的醚键的伸缩振动; 在 1686 与 2751 $\mathrm{cm}^{-1}$ 处还出现了醛基特征吸收, 前者为羰基 $\mathrm{C}=\mathrm{O}$ 的伸缩振动吸收, 后者为醛基上 $\mathrm{C}-\mathrm{H}$ 键的伸缩振 动吸收; 另外还显示出苯环的特征吸收峰(下面将分 析) 以及 $840 \mathrm{~cm}^{-1}$ 处二元取代后苯环上 $\mathrm{C}-\mathrm{H}$ 键的 面外弯曲振动(键合的苯甲醛). 上述这些谱峰的变 化充分表明, 对羟基苯甲醛已与 $\mathrm{P}$ (GMA-co-MMA) 侧链的环氧基团发生了开环成醚反应, 形成了键合 有 BA 的聚合物 BA-P(GMA-co-MMA).

在 PP-P(GMA-co-MMA)的红外光谱图中, 醛基 的特征吸收峰消失, 但却清楚地显现出了苯基吓啉 的诸特征吸收. 吓啉环的特征吸收出现在 $1350 、 960$ 及 $798 \mathrm{~cm}^{-1}$ 等处, 其中 1350 和 $798 \mathrm{~cm}^{-1}$ 处的峰为叶 啉环骨架伸缩振动吸收, $960 \mathrm{~cm}^{-1}$ 则为吓啉内环 $\mathrm{N}-\mathrm{H}$ 键的伸缩振动. 苯基卟啉外环上苯基的特征 吸收峰: $1600 \mathrm{~cm}^{-1}$ 处的苯环骨架 $(\mathrm{C}-\mathrm{C})$ 振动吸收; 3058 与 $3023 \mathrm{~cm}^{-1}$ 处的苯环上 $\mathrm{C}-\mathrm{H}$ 键的伸缩振动 吸收, 1504 及 $1450 \mathrm{~cm}^{-1}$ 处苯环上 $\mathrm{C}-\mathrm{H}$ 键的面内 及面外弯曲振动吸收; 762 和 $698 \mathrm{~cm}^{-1}$ 处的峰则为 苯环的单取代特征吸收. 上述这些谱峰的变化清楚 地表明, BA-P(GMA-co-MMA)、小分子苯甲醛与吡
咯之间已经发生了 Adler 反应, 形成了侧链键合有 卟啉的聚合物 PP-P(GMA-co-MMA).

\subsubsection{PP-P(GMA-co-MMA)的 ${ }^{1} \mathrm{H}-\mathrm{NMR}$ 谱}

图 2 给出了 PP-P(GMA-co-MMA) 的 ${ }^{1} \mathrm{H}-\mathrm{NMR}$ 谱. 由于在 $\mathrm{P}(\mathrm{GMA}-c o-\mathrm{MMA})$ 大分子链的侧链键合 了吓啉, 故在 PP-P(GMA-co-MMA)的 ${ }^{1} \mathrm{H}$ NMR 谱中 除显示出 $\mathrm{P}(\mathrm{GMA}-c o-\mathrm{MMA})$ 的各种氢质子的化学位 移外, 也清楚地显示出 PP 的各氢质子的化学位移. 在 PP-P(GMA-co-MMA)的大分子链中, 并非全部的 GMA 单元其侧链都键合了 PP, 还有少量的 GMA 单元未参加反应, 其侧链仍以环氧键的形式存在, 为 便于 ${ }^{1} \mathrm{H}-\mathrm{NMR}$ 谱的分析, 在下面的 PP-P(GMA-coMMA) 结构表达式中, 特将此少量未参加反应的 GMA 单元也表示出来.

$\mathrm{P}(\mathrm{GMA}-\mathrm{co}-\mathrm{MMA})$ 的各种氢质子的化学位移为 $\delta=1.044 、 1.218(\mathrm{~b})$ 处的两峰均为PP- P(GMA-co-MMA) 主链上侧甲基氢质子的特征共振信号, $\delta=1.900-$ 2.026(c)范围的两峰为主链上亚甲基氢质子的特征 共振吸收, $\delta=3.787(\mathrm{~g})$ 处的峰是 MMA 单元酯甲基的 特征共振信号, $\delta=2.730 、 2.893 、 3.542(\mathrm{e}, \mathrm{f})$ 处的峰为 GMA 单元未参加反应的环氧基团中氢质子的特征 共振吸收, $\delta=4.247(\mathrm{j})$ 处的两峰为 GMA 单元酯基中 亚甲基氢质子的特征共振信号, $\delta=3.895(\mathrm{~h})$ 处的两峰 为环氧键开环后次甲基氢质子的特征共振吸收, $\delta=$ 4.004(i)处的两峰为环氧键开环后醚键亚甲基氢质 子的特征共振信号, $\delta=2.323(\mathrm{~d})$ 处的两峰为环氧键开 环后仲羟基的氢质子特征共振吸收.

键合在 P(GMA-co-MMA) 侧链上的 PP 其诸氢 质子的化学位移: $\delta=-2.935$ (a) 的单峰为吓啉内环两 个与氮原子相连接的质子的共振信号, $\delta=7.147-$ $7.526(\mathrm{k})$ 的多重峰为 4 个苯环上间、对位质子的共振

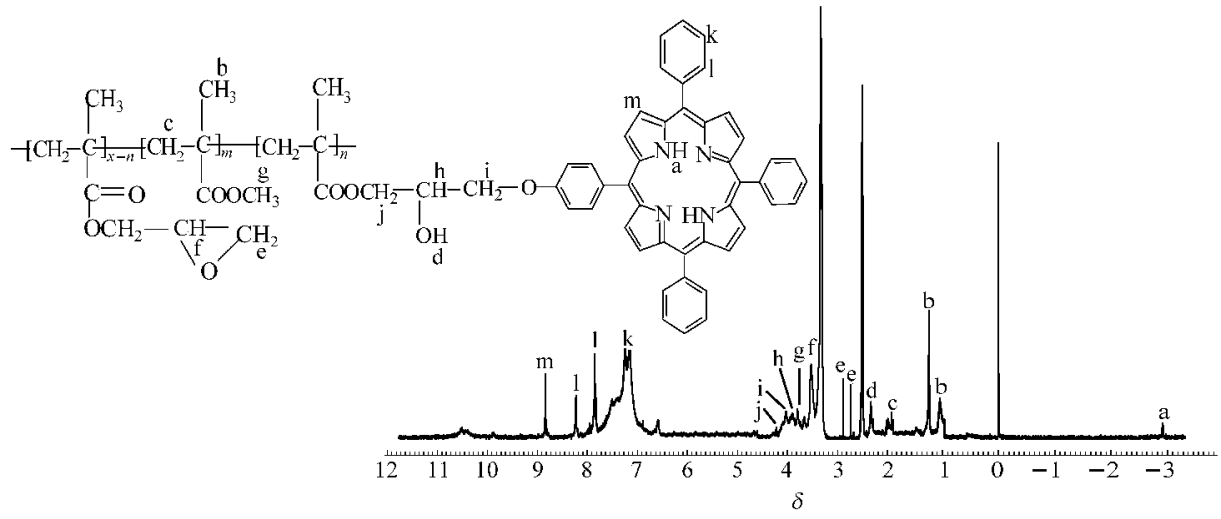

图 2 PP-P(GMA-co-MMA) 的 ${ }^{1} \mathrm{H}$ NMR 谱图

Fig.2 ${ }^{1}$ H-NMR spectrum of PP-P(GMA-co-MMA) 
信号, $\delta=7.852 、 8.204(1)$ 的多重峰为 4 个苯环上 8 个 邻位质子的共振信号; $\delta=8.829(\mathrm{~m})$ 的单峰是 4 个吡 咯环上 8 个质子的共振信号.

上述 ${ }^{1} \mathrm{H}-\mathrm{NMR}$ 谱数据进一步证实, 键合有苯甲 醛的大分子 BA-P(GMA-co-MMA)、小分子苯甲醛 与吡咯已经发生了 Adler 反应, 实现了吓啉的同步 合成与在大分子上的键合, 形成卟啉功能化的聚合 物 PP-P(GMA-co-MMA).

\subsection{PP-P(GMA-co-MMA)的电子吸收光谱}

使用 PP 键合量不同的 PP-P(GMA-co-MMA)样 品, 配制聚合物浓度相同的DMF溶液 $\left(C=0.03 \mathrm{~g} \cdot \mathrm{L}^{-1}\right)$, 图 3 给出了各聚合物的电子吸收光谱. 从图中看到, 由于在 $\mathrm{P}(\mathrm{GMA}-c o-\mathrm{MMA})$ 大分子链的侧基上键合了 卟啉, 使之也具有了卟啉的吸收性能. PP-P(GMA$c o-\mathrm{MMA})$ 的电子吸收光谱与文献报道 ${ }^{[17]}$ 的苯基卟啉 的电子吸收光谱相一致, $416 \mathrm{~nm}$ 附近的强吸收是 Soret 吸收带, 是叶啉分子的 $\pi$ 电子发生向第二电 子激发态跃迁 $\left(\pi-\pi^{*}\right)$ 所产生的; 在 $513 、 549 、 593$ 及 $645 \mathrm{~nm}$ 附近的弱吸收带是 $Q$ 吸收带, 是卟啉分子的 $\pi$ 电子发生向第一电子激发态跃迁 $\left(\pi-\pi^{*}\right)$ 所产生的.

从图 3 还可以看到, 随着 PP-P(GMA-co-MMA) 分子链中吓啉键合量的增大, 各吸收峰的强度逐渐 增强, 充分显示出大分子 P(GMA-co-MMA) 卟啉功 能化后的特征.

\subsection{PP-P(GMA-co-MMA) 的荧光发射光谱}

吓啉是具有双苂光行为的大环共轭化合物, 第 二单重激发态 $\left(S_{2}\right)$ 向基态 $\left(S_{0}\right)$ 发生 $S_{2} \rightarrow S_{0}$ 跃迁所产生 的苂光发射约在 $440 \mathrm{~nm}$ 附近, 这一发射带相应于 吸收光谱中的 Soret 带, 故被称为 Soret 发射带; 第 一单重激发态 $\left(S_{1}\right)$ 向基态 $\left(S_{0}\right)$ 发生 $S_{1} \rightarrow S_{0}$ 跃迁所产生

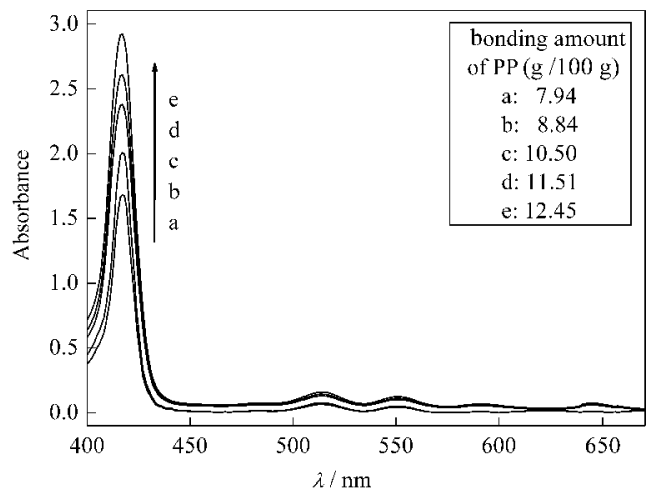

图 3 共聚物 PP-P(GMA-co-MMA) 在 DMF 溶液中的 电子吸收光谱

Fig.3 Electronic absorption spectra of PPP(GMA-co-MMA) in DMF solution

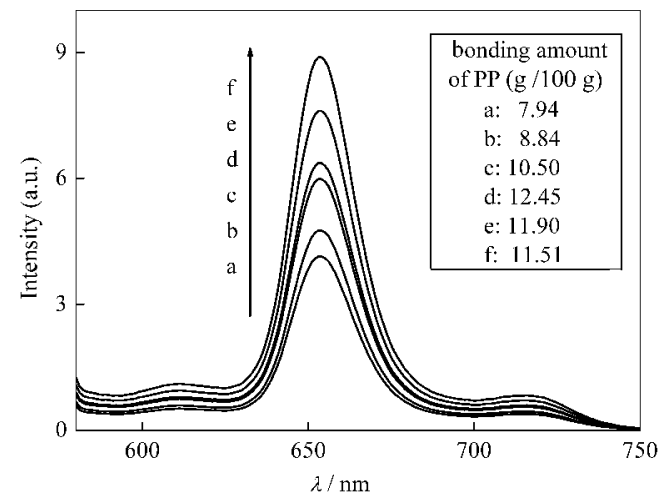

图 4 共聚物 PP-P(GMA-co-MMA) 在 DMF 溶液中的 荧光发射光谱

Fig.4 Fluorescence emission spectra of PP-P(GMAco-MMA) in DMF solution room temperature; excitation at $560 \mathrm{~nm}$

的苂光发射约在 $650 \mathrm{~nm}$ 附近, 相应于吸收光谱中 的 $Q$ 带, 因此被称为 $Q$ 发射带. 由于 $S_{2} \rightarrow S_{1}$ 的内转 化速度很快 ${ }^{[18]}$, 致使卟啉的 Soret 苂光带很弱, 因此 本文主要讨论 $Q$ 发射带.

以DMF为溶剂, 使用PP键合量不同的PP-P(GMAco-MMA)样品, 配制聚合物浓度相同的溶液, 图 4 给出了各聚合物的荧光发射光谱. 从图 4 可以看到, 由于在 $\mathrm{P}(\mathrm{GMA}-\mathrm{co}-\mathrm{MMA})$ 大分子链的侧基键合了卟 啉, 使之也具有了吓啉的光物理行为, 在 $650 \mathrm{~nm}$ 附 近产生了苯基吓啉的 $Q$ 发射带 ${ }^{[19]}$. 从图 4 还可以发 现, 随着 PP-P(GMA-co-MMA)分子链中吓啉键合量 的增大, $Q$ 带的发射带的强度呈现先增强后减弱的 变化规律. 为更清楚地显示上述规律, 由图 4 的数据 绘制苂光发射强度一卟啉键合量的关系曲线, 示于 图 5.

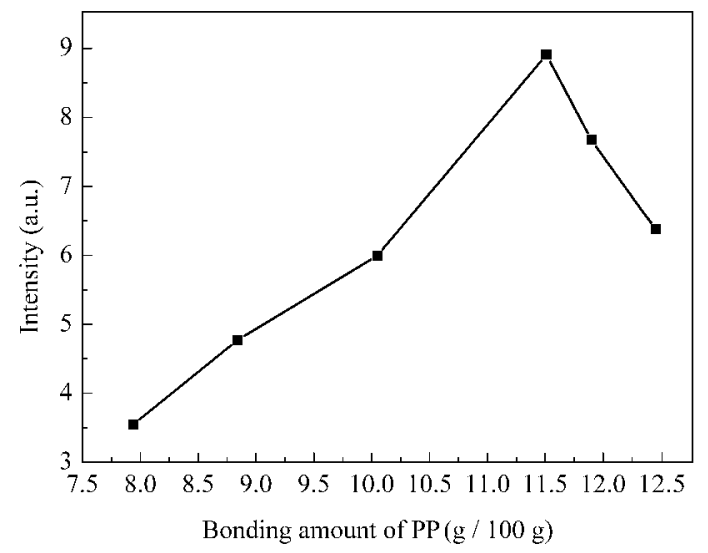

图 5 共聚物 PP-P(GMA-co-MMA) 荧光发射强度随 PP 键合量的变化曲线

Fig.5 Variation of fluorescence intensity with bonding amount of PP for PP-P(GMA-co-MMA) 


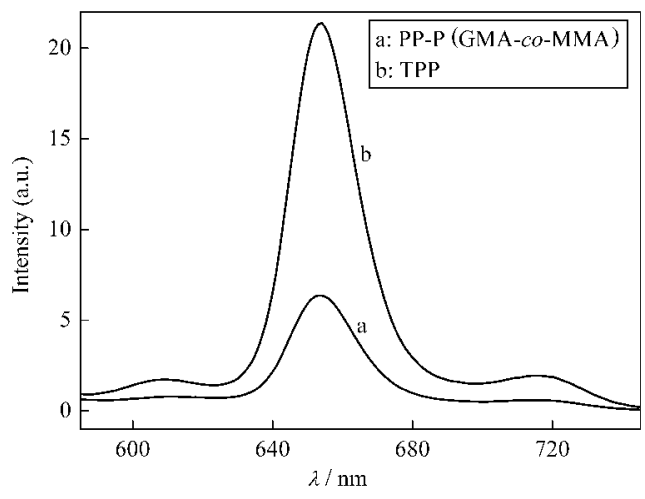

图 6 四苯基卟啉与聚合物 PP-P(GMA-co-MMA) 的 荧光发射光谱

Fig.6 Fluorescence emission spectra of TPP and copolymer PP-P(GMA-co-MMA)

room temperature; excitation at $560 \mathrm{~nm}$

当 PP-P(GMA-co-MMA)大分子链中卟啉键合量 较低时, 随着键合量的增大, 由于体系中发射苂光的 卟啉单元的浓度增大, 故 $Q$ 发射带的强度增强; 但 是随着卟啉键合量的进一步增大, 大分子 $\mathrm{P}(\mathrm{GMA}-$ $c o$-MMA)侧链上键合的吓啉单元密度进一步增大, 当卟啉单元密度增大到一定程度时, 同一条大分子 链中相近的吓啉侧基之间可能会发生能量转移 ${ }^{[20,21]}$, 产生苂光猝灭, 或形成激基缔合物, 从而导致在苂光 强度曲线上出现最高点后又转而下降的变化规律; 此后, 随着卟啉键合量的继续增大, 大分子 $\mathrm{P}(\mathrm{GMA}-\mathrm{co}-$ MMA)侧基的卟啉密度继续增大, 能量转移导致的 苂光猝灭程度加重. 显然, 上述实验结果反映出了叶 啉功能化大分子的一种高分子效应.

将图 5 中键合量最大的聚合物(卟啉键合量为 $12.45 \mathrm{~g} / 100 \mathrm{~g}$ )的苂光发射光谱与四苯基卟啉的菼光 发射光谱(两者溶液中吓啉单元的浓度均为 $7 \times 10^{-6}$ $\mathrm{mol} \cdot \mathrm{L}^{-1}$ ) 进行比较(见图 6), 苂光猝灭现象会显示得 更加清楚. 从图 6 的数据可以看出, 小分子卟啉 TPP 的 $Q$ 发射带强度 (21.31) 是 PP-P(GMA-co-MMA) 强 度(6.36)的 3.4 倍之多, 说明 PP-P(GMA-co-MMA)发 生了较强的苂光猝灭现象.

\section{3 结 论}

通过共聚物 $\mathrm{P}(\mathrm{GMA}-c o$-MMA)侧基环氧基团与 对差基苯甲醛之间的开环成醚反应, 形成了键合有 苯甲醛的大分子BA-P(GMA-co-MMA), 接着在溶液 体系中, 使小分子苯甲醛、BA-P(GMA-co-MMA)与 吡咯发生Adler反应, 实现了卟啉在大分子 $\mathrm{P}(\mathrm{GMA}$ co-MMA)侧链的同步合成与键合, 成功地制备了卟
啉功能化的线型聚合物 PP-P(GMA-co-MMA). 该叶 啉功能化的聚合物具有四苯基卟啉的光物理行为, 且电子吸收光谱强度随着卟啉键合量的增大而增 强. 其苂光发射光谱强度随着卟啉键合量的增大呈 现先增大后减小的变化规律, 是由于大分子链内发 生了能量转移的苂光猝灭所致.

\section{References}

1 Venosa, G. D.; Fukuda, H.; Batlle, A.; MacRobert, A.; Casas, A. Journal of Photochemistry and Photobiology B: Biology, 2006, 83: 129

2 Zhou, H.; Baldini, L.; Hong, J.; Wilson, A. J.; Hamilton, A. D. Journal of the American Chemical Society, 2006, 128: 2421

3 Ohmori, Y.; Itoh, E, Miyairi, K. Thin Solid Films, 2006, 499: 369

4 Moreira, M. S. M.; Martins, P. R.; Curi, R. B.; Nascimento, O. R.; Iamamoto, Y. Journal of Molecular Catalysis A, 2005, 233: 73

5 Liu, J. H.; Itoh, J. I. Talanta, 2006, 70: 791

6 Weng, Y. Q.; Teng, Y. L.; Yue, F.; Zhong, Y. R.; Ye, B. H. Inorganic Chemistry Communications, 2007, 10: 443

7 Takechi, K.; Shiga, T.; Motohiro, T.; Akiyama, T.; Yamada, S.; Nakayama, H.; Kohama, K. Solar Energy Materials \& Solar Cells, 2006, 90: 1322

8 Mukherjee, M.; Ray, A. R. Journal of Molecular Catalysis, 2007, 266: 207

9 Moghadam, M.; Tangestaninejad, S.; Mirkhani, V.; MohammadpoorBaltork, I.; Kargar, H. Bioorganic \& Medicinal Chemistry, 2005, 13: 2901

10 Grenoble, S.; Gouterman, M.; Khalil, G.; Callis, J.; Dalton, L. Journal of Luminescence, 2005, 113: 33

11 Lo, S. C.; Burn, P. L. Synthetic Metals, 1999, 102: 1089

12 Monnereau, C.; Blart, E.; Montembault ,V.; Fontaine, L.; Odobel, F. Tetrahedron, 2005, 61: 10113

13 Oh, K. C.; Lim, S. M.; Paeng, I. R.; Paeng, K. J. Journal of Electroanalytical Chemistry, 2001, 506: 42

14 Sendhil, K.; Vijayan, C.; Kothiyal, M. P. Optical Materials, 2005, 27: 1606

15 Górski, Ł.; Meyerhoff, M. E.; Malinowska, E. Talanta, 2004, 63: 101

16 Stevens, N.; Akins, D. L. Sensors and Actuators B, 2007, 123: 59

17 Zhang, P. Y.; Zhang, J. B.; Yu, X. C.; Zhang, L. W.; Wei, X. H. Acta Phys. -Chim. Sin., 2008, 24(1): 143 [张鹏燕, 张建斌, 于熙 昌, 张凌伟, 魏雄辉. 物理化学学报, 2008, 24(1): 143]

18 Deda, M. L.; Ghedini, M.; Aiello, I.; Franco, I. D. Inorganic Chemistry Communications, 2004, 7: 1273

19 Shi, Y. Y.; Zheng, W. Q.; Li, X. Q.; Yu, L. X.; Wang, X. Q. Chemical Journal of Chinese Universities, 2005, 26: 9 [石荣岩, 郑文琦, 李向清, 于连香, 王杏乔. 高等学校化学学报, 2005, 26: 9]

20 Balasubramaniam, E.; Natarajan, P. Journal of Photochemistry and Photobiology A: Chemistry, 1997, 103: 201

21 Nowakowska, M.; Karewicz, A.; Loukine, N.; Guillet, J. E. Polymer, 2002, 43: 2003 